\title{
EL ESQUEMA CRUZADO COMO FORMA DE ANÁLISIS CUALITATIVO EN CIENCIAS SOCIALES
}

THE CROSSED DIAGRAM AS A WAY OF QUALITATIVE ANALYSIS IN SOCIAL SCIENCES

Dr. Javier Corvalán (jcorvala@cide.cl) Centro de Investigación y Desarrollo de la Educación, Universidad Alberto Hurtado (Santiago, Chile)

\begin{abstract}
This article analyses the epistemological and methodological bases of crossed diagram in the field of social sciences and especially in qualitative research, considering that the origin of this diagram is located in the Cartesian coordinate system. Such coordinates provide elements that are at the bases of analytic geometry and from it, we highlight three aspects that have been 'exported' in the use of this diagram by the social sciences: the logic of the opposites; 'spacialisation'; and conceptual fecundity. From these three aspects, we analyse the contribution of structural linguistics and anthropological structuralism to this diagram, particularly regarding the goal of formalization in the social sciences. The article shows some of the uses given to this diagram by different contemporary authors, to end classifying those authors according to their analytical integration of epistemic and methodological aspects implicit in the Cartesian coordinate system.
\end{abstract}

Key words: qualitative, methodology, structural analysis, formalisation, Cartesian.

\section{Resumen}

El artículo analiza los fundamentos epistemológicos y metodológicos del esquema cruzado en el campo de las ciencias sociales y en particular del análisis cualitativo, considerando que el origen de tal esquema se encuentra en las coordenadas cartesianas. Tales coordenadas tienen un conjunto de elementos que a la vez están en la base de la geometría analítica y a partir de ella se resaltan tres aspectos que han sido "exportados" mediante el uso de este esquema, hacia las ciencias sociales: la lógica de opuestos, la "espacialización" o "espacialidad" y la fecundidad conceptual. A partir de esta triada de elementos se analiza el aporte de la lingüística estructural y del estructuralismo antropológico en torno al esquema en cuestión y en relación al objetivo de formalización en ciencias sociales. El texto ilustra algunos usos del esquema analizado realizados por distintos autores contemporáneos y termina por clasificar a los mismos de acuerdo a la integración, en tales análisis, de elementos epistémico-metodológicos implícitos en las coordenadas cartesianas.

Palabras clave: cualitativo, metodología, análisis estructural, formalización, cartesiano.

Introducción

Una de las formas más comúnmente utilizadas para la exposición y análisis de información cualitativa en ciencias sociales es el esquema cruzado o de coordenadas cartesianas. Por tal se entiende a una 
herramienta metodológica-conceptual que permite la ubicación y la oposición de conceptos a partir de combinatorias de tipo lógico. Aun cuando su origen como estructura de análisis se encuentra fuera de las ciencias sociales y se ubica, como veremos, en torno a la matemática y la lingüística, el esquema cruzado ha sido un instrumento utilizado de manera diversa y recurrente por científicos sociales de diversas orientaciones teóricas, por lo general, como parte de un proceso de ordenamiento y generación conceptual. No obstante, tal utilización no se ha estructurado en torno a procedimientos estandarizados y en consecuencia el uso que se ha hecho de esta herramienta ha sido disímil. En este artículo buscamos analizar los principios epistemológicos y metodológicos que están presentes en este esquema y las eventuales potencialidades que ofrece para efectos de la conceptualización en ciencias sociales (1).

\section{Algunos ejemplos de utilización}

La recurrencia y diversidad con la que esta herramienta metodológica ha sido utilizada queda reflejada en los siguientes ejemplos (2):

1) El sociólogo francés Alain Touraine, a propósito de una conceptualización de lo que llama los puntos cardinales de la sociedad (1978:103) utiliza el esquema para construir el campo de la sociología dando cuenta de sus cuatro elementos: acción, orden, crisis y cambio. En los ejes, Touraine sitúa un conjunto de conceptos que, de acuerdo a lo que señala en el texto, tienen una temporalidad y una consecuencialidad desde el exterior hacia el interior del eje respectivo. No queda claro que los conceptos puntas de los ejes estén definidos como opuestos, ni tampoco que tales conceptos den lugar a nuevas conceptualizaciones en cada campo, lo que más adelante llamaremos fecundidad conceptual. El esquema propuesto es el siguiente:

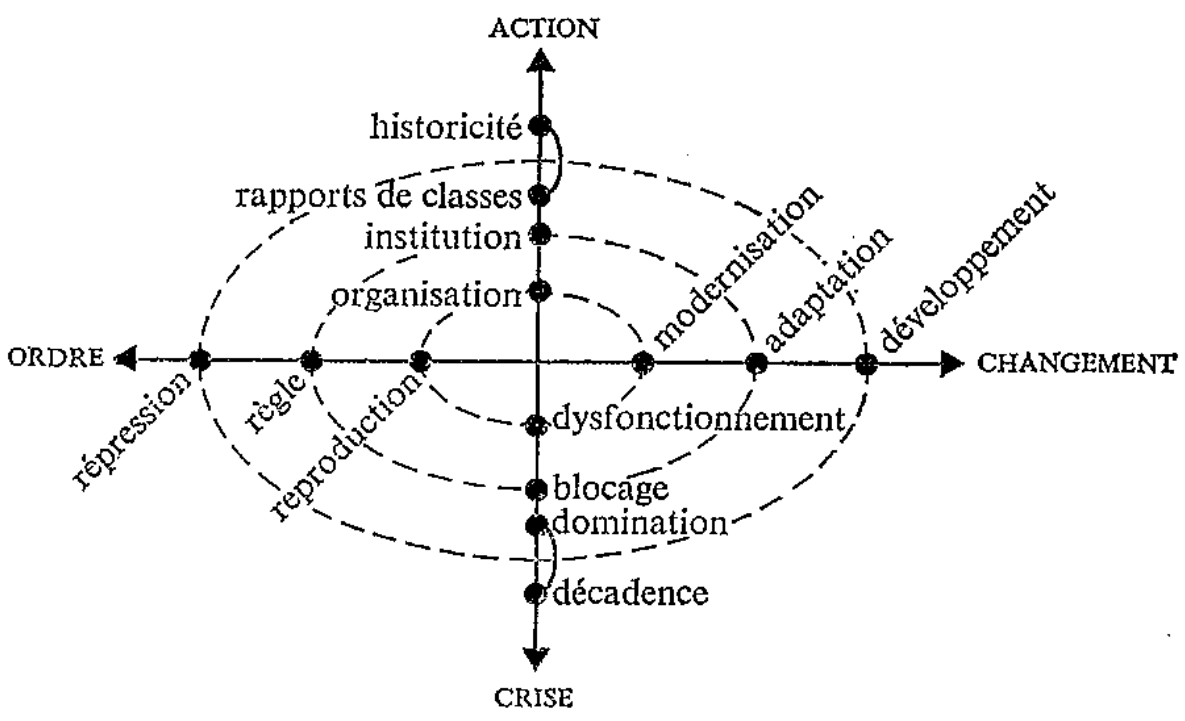

Fuente: Touraine 1978:103.

2) El mismo Touraine en 1981 utiliza el esquema a propósito de un estudio sobre el movimiento nacionalista occitano en el sur de Francia, dentro de una línea mayor de análisis sobre los movimientos sociales en las sociedades contemporáneas. El esquema es el siguiente: 
Fuente: Touraine 1981:222.

En este esquema los autores establecen "dos ejes que definen el populismo: el que une identidad y cambio y el que une clase y cambio" (Touraine et. al. 1981:212). Como se trata de una investigación que se realiza desde la perspectiva accionalista y con la metodología de la intervención sociológica, los autores proponen el esquema y los individuos estudiados se ubican ellos mismos en él. Los autores se basan en un marco teórico original de la sociología de los movimientos sociales. Los conceptos polares del esquema cruzado utilizado surgen en el momento de proponer dos hipótesis concretas aplicadas para el caso del nacionalismo occitano: "la primera definió el movimiento occitano como una acción colectiva orientada hacia el control del cambio más que a salvaguardar la identidad. La segunda da más importancia a una lucha propiamente social contra los dominadores de hoy en día, comerciantes y especuladores pero también turistas y el ejército apropiándose de tierras. Esta hipótesis renuncia a ver en el movimiento occitano una lucha por la orientación del cambio histórico" (Touraine et. al. 1981:173).

3) George Ritzer, sociólogo norteamericano contemporáneo, propone una tipología para clasificar formas de análisis sociológico también sobre la base de conceptos en oposición. En este caso, Ritzer considera los dos ejes de su esquema como elementos explícitamente en oposición los que, además, representan una continuidad conceptual. El origen de tal conceptualización -reconoce Ritzer- pertenece al sociólogo rusofrancés Georges Gurvitch quien, en 1964, formula un complejo esquema (al decir de Ritzer) para dar cuenta de los niveles de análisis sociológico, utilizando los cuatro conceptos matrices considerados más tarde por Ritzer: macroscópico/microscópico y objetivo/subjetivo. Resulta entonces interesante que el esquema cruzado de Ritzer se proponga como una clarificación de la complejidad indicada por Gurvitch: "el trabajo de Gurvitch plantea una dificultad importante: su esquema analítico es extremadamente complejo y confuso (...) el mundo social es muy complejo, y para poder comprenderlo, se requieren modelos relativamente simples" (Ritzer 1996:609). Por otro, lado, Ritzer aprovecha el esquema cruzado en uno de sus elementos centrales, tal como lo explicaremos más adelante: la fecundidad conceptual a la cual el autor le otorga un rol central: "aunque estos dos continua son harto interesantes, lo que nos concierne aquí es la interrelación entre ambos" (Ritzer 1996:610). El esquema es el siguiente: 


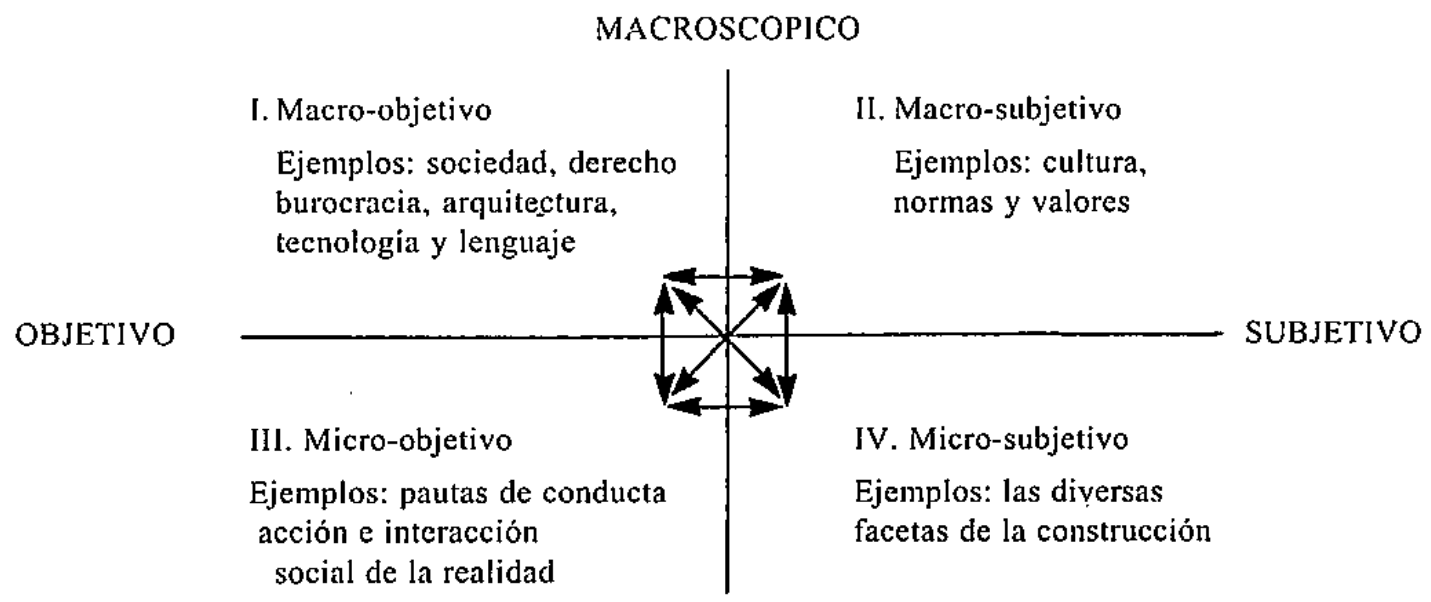

MICROSCOPICO

Fuente: Ritzer 1996:610.

4) José Joaquín Brunner, investigador chileno, recurre reiteradas veces al esquema en cuestión al tratar temas distintos tales como los modos culturales propios de las sociedades modernas (Brunner 1994), la globalización cultural (Brunner 1998) y las dinámicas de transformación de los sistemas de educación superior (Brunner 2000), utilizando una veces más que otras una presentación de datos opuestos. A manera de ejemplo tomaremos solo un texto de este autor (1994). En él, Brunner usa reiterativamente el esquema cruzado principalmente con el fin de generar lo que más adelante llamaremos "fecundidad conceptual". El autor aborda el concepto de modos culturales en las sociedades contemporáneas y en las primeras páginas de su texto realiza una suerte de justificación metodológica de los esquemas cruzados a seguir, todo ello en torno a la noción de bidimensionalidad del análisis: "la sociología, en general, y la sociología de la cultura, en particular, han tendido a enfrentar su problemática, sin embargo, de manera unidimensional (...) particularmente en el campo de los estudios culturales, los intentos por producir una síntesis bidimensional son escasos y frustrantes (...) una posibilidad de enfocar bidimensionalmente estas cuestiones que aquí deseamos explorar consiste en distinguir los modos culturales que resultan de combinar los ejes de la acción y el control" (Brunner 1994:11). A partir de esta declaración de profundización analítica, el autor desarrolla tres esquemas seguidos que parten desde dos oposiciones: control y acción, generando ellas dos polaridades respectivas: individuo y grupo y jerarquía y autonomía. La combinación (o fecundidad) de esta conceptualización polar genera cuatro "modos culturales puros" (selectivo, competitivo, comunitario y ritual) (Brunner 1994:16), cada una de los cuales, a su vez genera "situaciones típicas" (escuela, mercado, amistad y estamento) para llegar a la combinatoria o fecundidad final de "seis modalidades culturales que resultan de la combinatoria de modos puros". El esquema es el siguiente: 


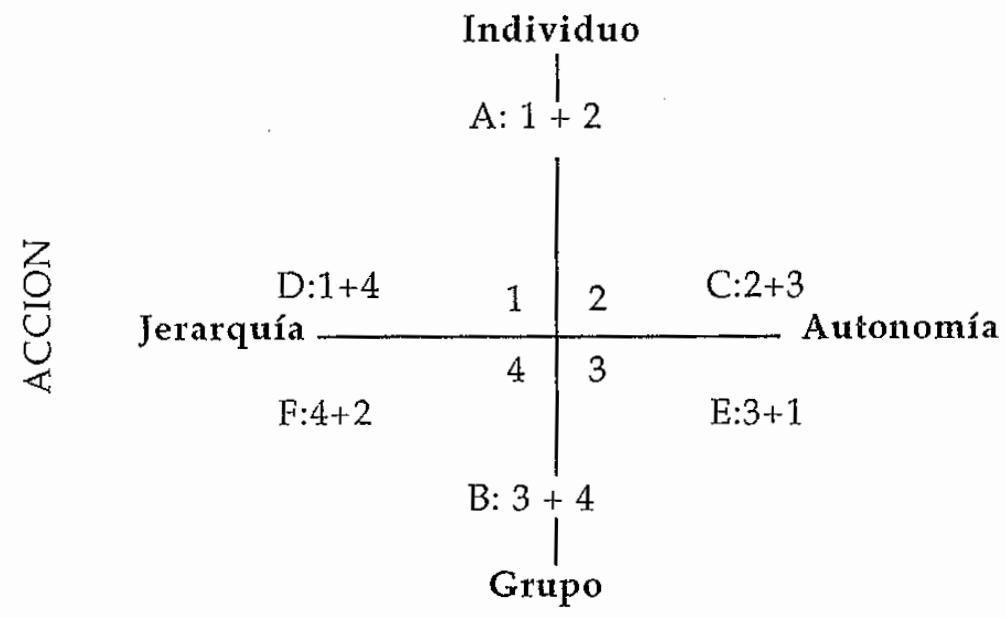

CONTROL

Fuente: Brunner 1994:20.

De lo anterior se desprende que Brunner recoge de las coordenadas cartesianas la lógica de oposición, la fecundidad conceptual, pero no la espacialidad, concepto que también examinaremos más adelante.

5) Un equipo de investigadores franco-chilenos (Dubet et. al. 1989) utiliza el esquema en cuestión para dar cuenta de las lógicas de acción colectiva de los pobladores chilenos. La investigación se plantea desde la teoría de los movimientos sociales (Touraine 1978) y el esquema resume, en sus conceptos polares, lo esencial de la teorización para explicar el fenómeno: “Exclusión y explotación, ruptura y participación, forman dos dimensiones independientes a partir de las cuales puede ser construido el sistema de acción de los pobladores. Basta con asociar las variables de la definición del actor y las de su relación al medio ambiente" (Dubet et. al. 1989:50). De acuerdo a lo señalado por los autores, el esquema se basa en dos conceptos de base: definición del actor y relación con el medio ambiente. Cada uno de estos conceptos tiene dos polaridades conceptuales en términos de oposiciones: explotación/exclusión y ruptura/participación. La combinatoria propuesta tiene fecundidad puesto que generan un concepto de acción colectiva en cada campo o cuadrante y a la vez un concepto de individuo y/o de actor colectivo. El esquema es el siguiente: 


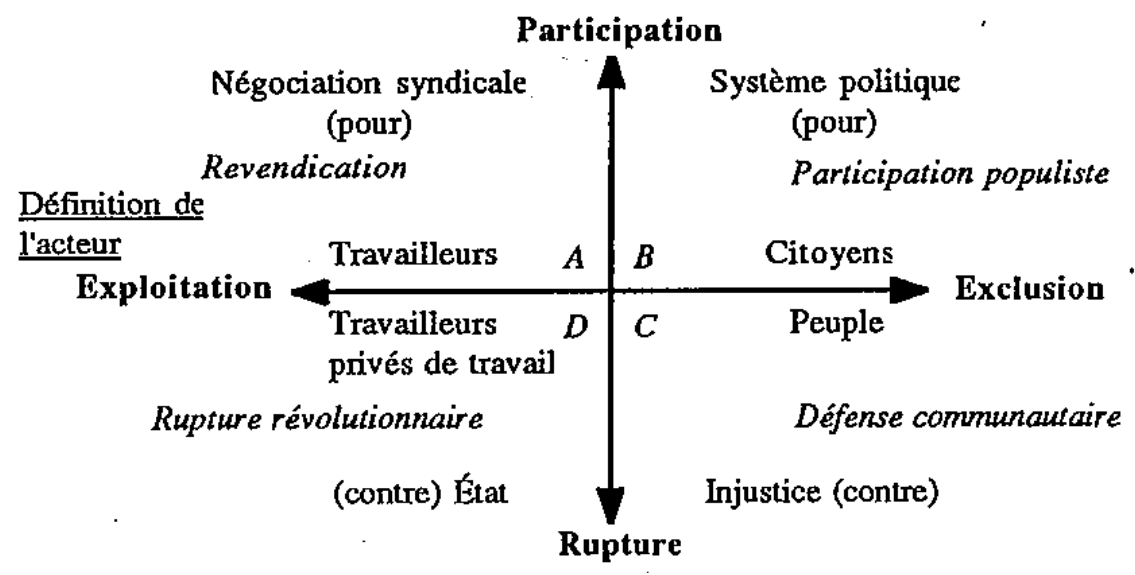

Fuente: Dubet et. al. 1989:50.

6) Por último el sociólogo francés Pierre Bourdieu representa el concepto de campo en un esquema cruzado que, en su teorización, corresponde a un espacio social (Bourdieu 1979:140). Varios son los elementos que Bourdieu recoge en su esquema y que provienen de las propiedades de las coordenadas cartesianas, siendo el primero el de la lógica de opuestos, que en su esquema sigue las relaciones de mayor y menor volumen de capital y, lo mismo en el otro eje, en relación al capital económico y al capital cultural. Es interesante que Bourdieu sitúa en el eje de la ordenada la cantidad global de capital que disponen individuos y grupos y en el de abscisa solo dos formas de este capital, el económico y el cultural, ya que considera que son las más eficaces para diferenciar prácticas sociales y estilos de vida en las sociedades contemporáneas (Bourdieu 2007:18) y, según esto, en un eje se condensa lo que está en otro. El segundo elemento que se condice directamente con los principios de las coordenadas cartesianas es el de espacialidad. Los fenómenos reseñados al interior del esquema (estilos de vida o categorías de ocupación) hacen que los agentes tengan "tantas cosas en común cuanto más próximos están en ambas dimensiones y tantas menos cuanto más alejados" (Bourdieu 2007:18). El tercer elemento del esquema de Bourdieu que recoge la lógica de las coordenadas cartesianas es menos evidente y se trata de la fecundidad. Como lo hemos visto en los autores anteriores, no necesariamente a partir de los cruces de cada concepto polar en las coordenadas se generan nuevos conceptos, lo que se debe a que al interior de cada dimensión se produce, según Bourdieu, una progresión (según lo dicho anteriormente) y no un fenómeno absoluto. El esquema que recoge una síntesis de la versión original es el siguiente: 


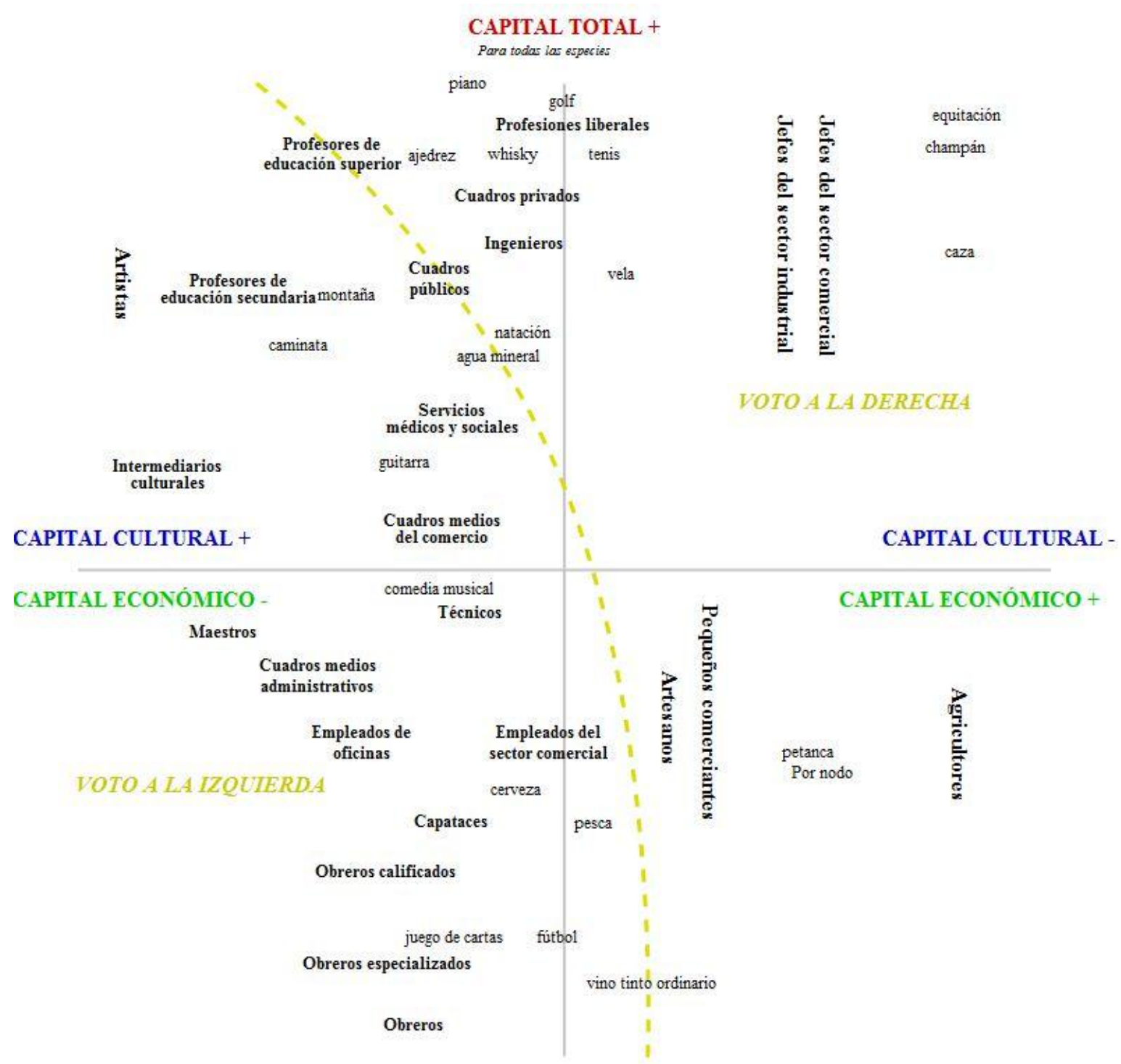

\section{CAPITAL TOTAL -}

Fuente: http://upload.wikimedia.org/wikipedia/commons/e/ed/Espace_social_de_Bourdieu-es.svg

Teniendo en cuenta la recurrencia del esquema cruzado en el análisis social, nos proponemos a continuación entregar algunos elementos históricos, metodológicos y epistemológicos respecto de este procedimiento de análisis, de manera de ayudar a comprender tanto las potencialidades como las limitaciones que presenta como instrumento de investigación social.

\section{El origen conceptual}

El llamado esquema cruzado tiene un origen en las llamadas coordenadas cartesianas, una estructura fundamental de la geometría analítica. Lo que poca gente sabe es que al parecer no fue René Descartes el creador de las coordenadas cartesianas: en su libro más fundamental en la materia "La géométrie" (Descartes 1858) no existe ningún esquema exactamente igual a como se conocen estas coordenadas 
actualmente y al tipo de análisis que nos ocupa en este artículo. En todo caso, hay dos elementos que son de consenso al respecto. El primero es que Descartes es uno de los principales creadores de la geometría analítica, rama de la matemática que va a tener en las coordenadas cartesianas uno de sus puntos de arranque más fuertes y que tal rama de la matemática moderna tiene como propósito la representación gráfica de relaciones algebraicas mediante algo que es propio de toda geometría: la ubicación de puntos en el espacio.

Este origen del esquema cruzado no deja de llamar la atención para el objetivo de este artículo, por cuanto se constata que un constructo netamente de origen cuantitativo resulta, siglos después, ampliamente utilizado en el ámbito de la investigación cualitativa y de la exposición conceptual en ciencias sociales, tal como lo demuestran los ejemplos anteriores. Es importante también señalar que parte importante de la estadística que se va a desarrollar en los siglos XIX y XX, en particular la de tipo inferencial, se basa en la utilización al menos parcial de las coordenadas cartesianas, centrando la mayor parte de su análisis en el cuadrante I de tales coordenadas, es decir, aquel que se corresponde con las cantidades positivas en ambos ejes. Los ejemplos más claros al respecto son tanto los análisis de correlación y regresión y más claramente, el análisis de correspondencia.

Por último, existe otro par de elementos, propios de las coordenadas cartesianas y por tanto de la geometría analítica, que van a ser de primera importancia para la posterior utilización en el campo de las ciencias sociales. El primero de ellos es la existencia de números o valores negativos, lo que se va a corresponder con el segundo elemento, la lógica de opuestos o de contrarios. Mientras el concepto de números negativos va ser operacionalizado en el campo de la matemática, la lógica de contrarios u opuestos va a ser parte de la reflexión en teoría del conocimiento, en lógica formal y en la lingüística estructural. Volveremos a este tema más adelante en tanto a continuación haremos una breve explicación de la estructura de las coordenadas cartesianas.

\section{Las coordenadas cartesianas}

Para entender lo que las coordenadas cartesianas representan y su posterior "exportación" a la metodología cualitativa en ciencias sociales, hay que partir considerando que la geometría analítica se propone ordenar valores numéricos en escalas que se crucen (o que se originen) en un punto de valor 0 o punto de origen. $\mathrm{A}$ partir de ese punto de origen se establecen dos líneas rectas o ejes en posición perpendicular a partir de las cuales los valores tienen progresión, ya sea positiva o negativa. Con ello cualquier punto puede tener una ubicación que será referenciada por una combinatoria de valores de los ejes. La representación más simple de lo señalado es lo siguiente: 


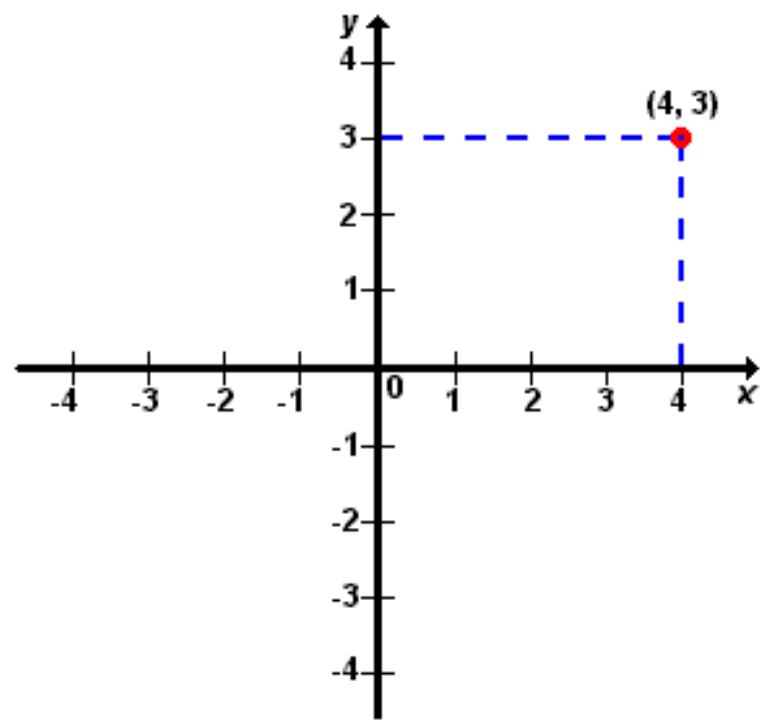

Fuente: http://willithanner.wordpress.com/2011/08/08/igualdad-de-parejas

En este sistema de coordenadas, el punto se ubica en la combinatoria del valor 3 del eje vertical y del valor 4 del eje horizontal, representándose $(4,3)$. En el sistema de coordenadas cartesianas, la línea horizontal recibe el nombre de abscisa y se representa por la letra $X$, en tanto la línea vertical se denomina ordenada y se representa por la letra Y. La letra que designa a cada uno de estos ejes va a ser importante para la disciplina estadística que se va a desarrollar siglos más tarde por cuanto denotan, en los análisis que implican causalidad (principalmente correlaciones y regresiones), una anterioridad temporal de los valores o variables indicados en la abscisa por sobre los de la ordenada, lo que se va a conocer también como variable independiente y variable dependiente.

Esta formalización da lugar a cuatro campos o cuadrantes:

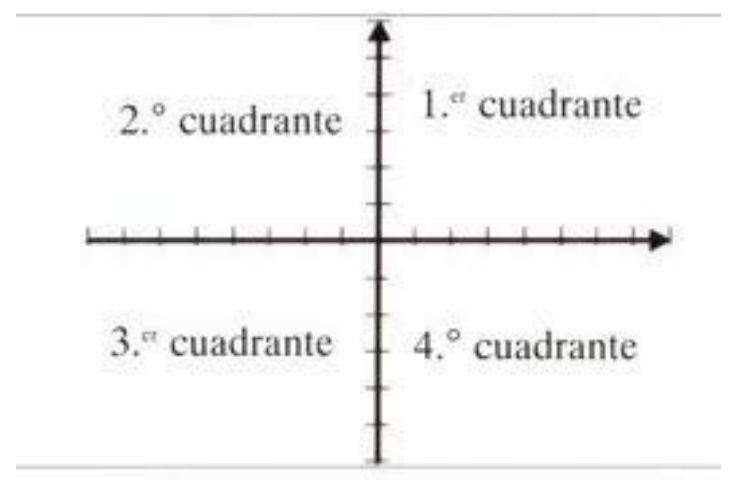

Fuente: http://ceipntrasradelapiedad.wordpress.com/2010/08/20/las-coordenadas-cartesianas 
Cada uno de los ejes, como señalamos, implica una secuencia de valores desde el punto cero que les da origen. A partir de este punto de origen, los valores indicados en ambas rectas aumentan o decrecen, de acuerdo a si son positivos o negativos y con ello cada campo resultante presenta una combinatoria particular de valores, como lo muestra el siguiente gráfico:

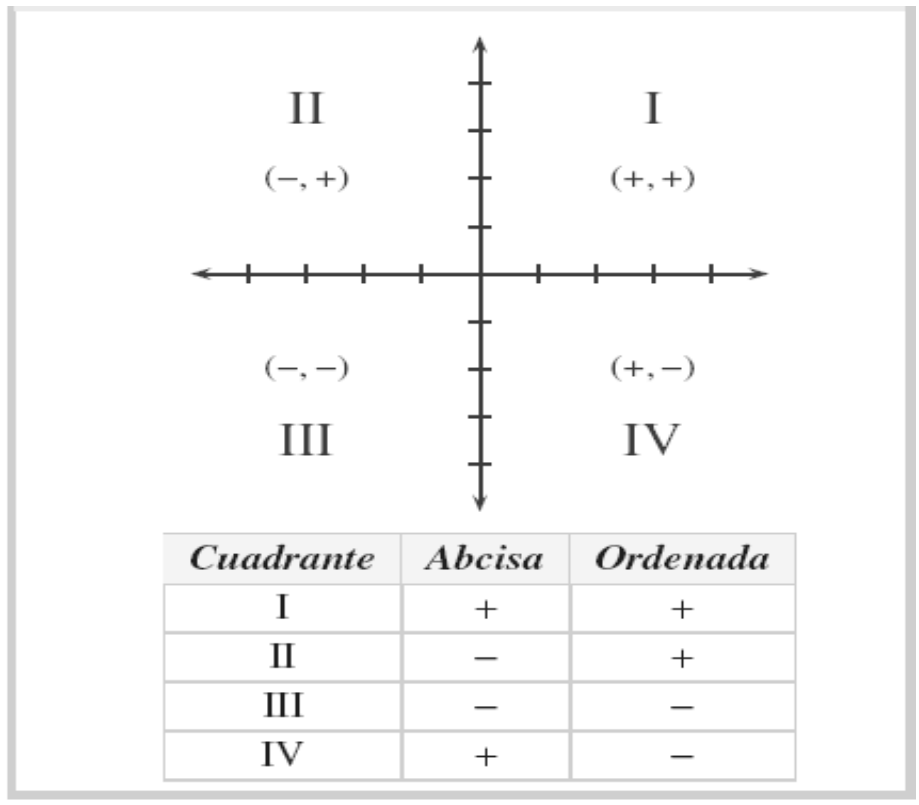

Fuente:

http://www.gobiernodecanarias.org/educacion/4/Medusa/GCMWEB/Code/Recursos/DetalleRecurso.aspx?। $\mathrm{dNodo}=263 \& \mathrm{IdRecurso}=8629 \&$ Preview $=\mathrm{Si}$

Lo anterior implica elementos que tendrán un símil o una misma epistemología en el análisis cualitativo y/o en la conceptualización en ciencias sociales, como por ejemplo que el campo I será el de los valores totalmente positivos (++), el campo II implica los valores de $X$ negativos y los de $Y$ positivos (- +), el campo III implica los valores de $X$ e $Y$ negativos (- -) y, por último, el campo IV implica los valores de $X$ positivos y los de Y negativos ( + -). De acuerdo a esto, hay un campo que es totalmente positivo (el I), otro que es totalmente negativo (el III) y dos que son mixtos, pero que en su composición no son iguales. De lo anterior se deduce también algo que ha sido la norma de la geometría analítica y es que el campo o cuadrante I es siempre el que es totalmente positivo y que la denominación numérica de cada cuadrante debe ser siempre la que hemos señalado en este artículo. En los ejemplos iniciales de utilización de esquemas cruzados se observa cómo algunos de los autores referidos no utilizan el orden señalado.

IV. De los valores numéricos a los conceptos cualitativos: La aplicación de las coordenadas cartesianas en investigación cualitativa en ciencias sociales

Para referirnos al paso de las coordenadas cartesianas al campo de la investigación cualitativa en ciencias sociales, llamaremos esquema cruzado a la utilización del mismo en tal tipo de investigación social. Lo importante, nos parece, es entender qué elementos pertenecientes a las coordenadas cartesianas se rescatan en el esquema cruzado, qué nuevos conceptos (tanto implícitos como explícitos) se incorporan y cuáles son los fundamentos epistémico-conceptuales en ambos casos. 


\section{La influencia de la lingüística y del estructuralismo}

Como es de amplio conocimiento, la investigación cualitativa ha pasado por diferentes etapas que, por lo general, pueden ser leídas desde una menor a una mayor exigencia de formalización. En particular la antropología estructural, bajo la influencia de Lévi-Strauss y esta a su vez inspirada en la lingüística estructural, son las que van a imponer la idea de la formalización dentro del análisis cualitativo, lo cual es posible a partir de la utilización de procedimientos estandarizados de análisis basados en la lógica formal que, a su vez, sirvan para explicar la existencia de lógicas de pensamiento y acción de los sujetos estudiados. En esta perspectiva de exigencia de formalización, el esquema cruzado aparece como un instrumento en extremo útil para la investigación cualitativa en ciencias sociales.

El surgimiento de gran parte del estructuralismo en ciencias sociales está fuertemente marcado, como señalamos en el párrafo anterior, por la lingüística estructural y el mismo Lévi-Strauss reconoce enfáticamente la influencia y modelización que la lingüística, como disciplina, realiza sobre el pensamiento estructuralista: "Dentro del conjunto de las ciencias sociales al que indiscutiblemente pertenece, la lingüística ocupa no obstante un lugar excepcional: ella no es más una ciencia social como las otras, sino aquella que, de lejos, ha realizado los más grandes progresos" (Lévi-Strauss 1974:43). Como lo cita y recuerda el propio Lévi-Strauss, este reconocimiento de lugar prominente y de modelo de la lingüística sobre otras ciencias sociales venía ya de Marcel Mauss quien señala: "la sociología estaría, claramente, mucho más avanzada, si ella hubiese procedido, en todo, a imitar a los lingüistas" (citado en Lévi-Strauss 1974:44). La especificidad de esta fascinación hacia la lingüística se manifiesta, en todo caso, no sobre todo tipo de lingüística, ni a todas sus dimensiones, sino esencialmente a la de tipo estructural y en particular a la definición de su método y a la conceptualización de su objeto de estudio. Ambos aspectos pueden resumirse en una palabra: formalización.

Lévi-Strauss define con claridad los aspectos que son propios del análisis lingüístico de tipo estructural y que deberían modelar las ciencias sociales estructuralistas: i) se concentra en el estudio de fenómenos inconscientes; ii) sus objetos de estudios son relacionales y no estructuras independientes; iii) introduce la noción de sistema; iv) busca leyes generales ya sea por inducción o por deducción (Lévi-Strauss 1974: 46).

El esquema cruzado, a nuestro parecer, recoge la mayor parte de estos principios. En primer lugar, vemos en los casos revisados que se trata de una conceptualización de realidades no necesariamente conscientes para los individuos, sino más bien constructos en los cuales ellos están inmersos (posicionamientos estructurales: los individuos y las instituciones que ellos integran no saben que son ubicables en una parte del campo conceptual). La excepción es el texto de Touraine et. al. (1981), ya que forma parte de una investigaciónacción. Aun así, los términos polares propuestos surgen de la lógica de investigación de los autores y no de los sujetos investigados.

En segundo lugar ( $y$ es el aspecto que nos parece más evidente), todos los autores utilizan el esquema en cuestión a partir de conceptos relacionales, es decir, de términos que se explican cada uno por una oposición a otro del mismo eje; tercero, el esquema cruzado implica y al mismo tiempo trata de conceptualizarse al interior de un concepto de sistema, tema que es propio de todo estructuralismo. Por sistema se entiende a un conjunto de elementos relacionados cuya interacción genera un tipo de comunicación cuya forma no es evidente (Lévi-Strauss 1974). En el caso de cada uno de los autores revisados, nos parece que la formalización realizada tiene un intento de clausura conceptual del sistema referido: la conceptualización que se hace de la realidad analizada incorpora esos y no otros conceptos; y 
cuarto, se trata de formalizaciones tendientes a generalizaciones, es decir, a explicaciones globales de fenómenos que se entienden por lógica de opuestos y por relación entre sus conceptos matrices.

\section{El esquema cruzado en el análisis estructural propiamente tal}

El desarrollo de la teoría estructuralista en ciencias sociales generó una teoría asociada a un método y este último particularmente con los aportes de Barthes (1971) y Greimas (1971), se consolidó en las décadas pasadas como un proceso estandarizado de análisis. La influencia más reciente ha sido en el campo del análisis sociológico cualitativo proveniente de autores de lengua francesa. Hiernaux (1977) y más tarde Piret et. al. (1996), además de Rémy (1990), han tratado de estandarizar los procedimientos más detallados del análisis estructural como método de análisis cualitativo. Precisamente, afirman estos autores, se trata de identificar dicotomías que conduzcan a formas estructuradas de organización del pensamiento y con ello de explicación de la acción social, proyecto para el cual es útil el esquema cruzado de base cartesiana.

Hiernaux (1977) es uno de los autores que más profundamente ha desarrollado el uso del método estructuralista en sociología, buscando una formalización del mismo a partir de los principios ya enunciados del paradigma estructuralista. A partir del análisis de disyunciones o binaridades, cuyas combinaciones forman estructuras mínimas de significado, llega a la posibilidad de los ejes cruzados y del llamado producto axial, que equivale al esquema cruzado. El avanzado nivel de formalización que propone queda expuesto en la siguiente gráfica:

représentation graphique (pour $\mathrm{n} \emptyset ; \mathrm{n}=2$ ) :

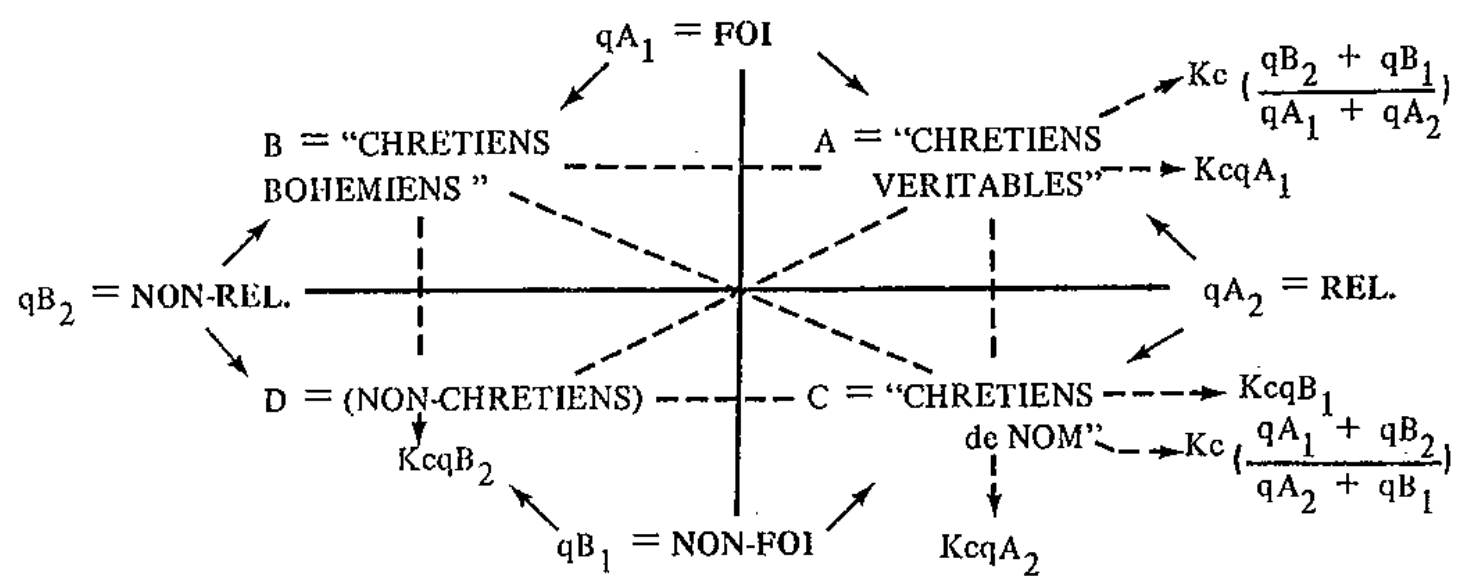

Fuente: Hiernaux 1977:56.

En ella se observan dos oposiciones: fe/no fe y religión/no religión, cuya combinatoria da lugar a cuatro tipos de cristianos: verdaderos, bohemos, no cristianos y cristianos de nombre. Tal como lo señala el autor: "si los cristianos verdaderos y los cristianos bohemios se homologan por la fe, los cristianos verdaderos se homologan además a los cristianos de nombre, por la religión y estos últimos se homologan a los no cristianos, por la falta de fe. Mientras que los no cristianos se homologan ellos mismos a los cristianos bohemios, por la falta de religión. La oposición fe/no fe separa así a los cristianos bohemios y los cristianos verdaderos, por un lado, de los cristianos de nombre y de los no cristianos, por otra parte, mientras que la 
oposición religión/no religión reagrupa a los cristianos verdaderos y los cristianos de nombre para oponerlos a los cristianos bohemios y a los no cristianos, en conjunto" (Hiernaux 1977: 55).

Piret, Nizet y Bourgeois (1996), consideran la propuesta metodológica de Hiernaux otorgándole un lugar central al producto axial, al que van a denominar estructura cruzada. Estos autores recogen la base de la explicación estructuralista argumentando que tal análisis, en un material cualitativo, consiste en la búsqueda de disyunciones o términos opuestos pero, a la vez, relacionados por un concepto de totalidad. Para estos autores, un mínimo de dos disyunciones forma una estructura. En ciertos casos, el orden del discurso que se analiza, organizado en disyunciones, es apto para las estructura tipo cruzado. Un ejemplo al respecto es el siguiente:

"Más vale una injustica que un desorden"

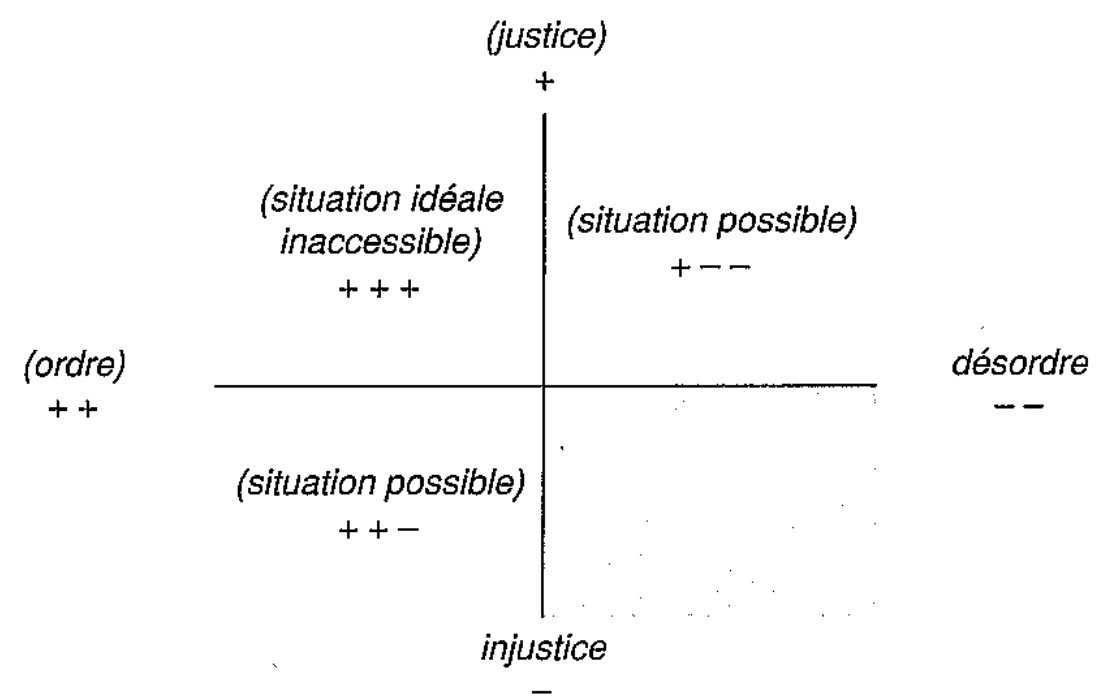

Fuente: Piret et. al 1996:75.

Este tipo de utilización del esquema cruzado conlleva, además de la lógica de opuestos (aun cuando nos parece que los autores, de acuerdo al ordenamiento original de las coordenadas cartesianas, debieron haber situado el polo positivo del eje horizontal en el lado derecho), otros elementos que en algunos casos van a ser recogidos en los textos de los autores que nos sirvieron de ejemplo al inicio de este artículo. Uno de ellos es la de fecundidad a partir de campos o realidad lógicamente posibles aun cuando no los sean aún en términos empíricos. Las realidades fecundadas, tal como las llaman Piret et. al. (1996), corresponde a las conceptualizaciones que surgen en cada uno de los campos del esquema y que se van a referir a realidades teóricamente posibles y/o a realidades consideradas explícitamente por los actores estudiados (Piret et. al. 1996:50). En el ejemplo anterior, las realidades fecundadas corresponden entonces a las situaciones señaladas al interior de cada cuadrante.

Otro aspecto es el de las valorizaciones y que dice relación con aspectos definidos como realidades negativas o poco deseables o, bien lo contrario, lo que se utiliza generalmente no en la explicitación de marcos teóricos a partir del esquema cruzado, sino en la interpretación de estructuras no manifestadas en los sujetos estudiados (Piret et. al. 1996:54). En el ejemplo en cuestión, las valorizaciones se encuentran en 
los términos polares orden/desorden y justica/injustica y en consecuencia en las realidades fecundadas en cada uno de los cuadrantes.

\section{La lógica de opuestos y sistema relacional}

Como ya lo mencionamos, la idea de lógica de opuestos proviene de dos tradiciones, lingüística y filosófica, sin referirnos a la tradición matemática a partir de los números positivos y negativos. Refiriéndonos solo a la primera, ella surge de la ya mencionada lingüística estructural en su línea más cercana a la semántica (bajo la figura de De Saussure) y de la fonología (bajo la figura Trubetzkoy). En ambos casos y tal como aludirá Greimas décadas más tarde, subyace la idea de continuidad y ruptura, pues tanto el significado como los sonidos de una lengua pertenecen a un sistema que opone cada uno de sus elementos, al mismo tiempo que los refiere a una unidad mayor: "Percibimos diferencias y, gracias a esta percepción, el mundo toma forma ante nosotros y para nosotros. Pero ¿qué significa exactamente -en el plano lingüístico- la expresión percibir diferencias?: 1. Percibir diferencias quiere decir que captar al menos dos términos-objeto como simultáneamente presentes. 2. Percibir diferencias quiere decir captar la elación entre los términos, vincularlos de una manera u otro (...) Dos consecuencias se desprenden inmediatamente de dicha definición: 1. Un solo término-objeto no conlleva significación; 2. La significación presupone la existencia de la relación: lo que es condición necesaria de la significación es la aparición de la relación entre dos términos" (Greimas 1971:28).

En el sistema, cada elemento adquiere su sentido y connotación en la medida que se opone a otro e incluso, a todos los demás que integran tal sistema. De esta manera, por ejemplo De Sausurre, en su Curso de Lingüística General, conceptualiza el objeto de estudio de la lingüística a partir de dos oposiciones o dicotomías básica: lengua/habla (cuya totalidad o referente mayor es el lenguaje) y significante/significado (cuya totalidad o referente mayor es el signo lingüístico). Trubetzkoy hace otro tanto cuando señala que "la fonología actual no se limita a declarar que los fonemas son siempre miembros de un sistema, ella muestra sistemas fonológicos concretos y pone en evidencia su estructura" (Trubetzkoy citado en Lévi-Strauss 1974:46).

Esta idea de unidad y diferencia, de continuidad y quiebre como manera de constituir conceptualmente lo real, está en la base de los postulados lingüísticos estructurales y de gran parte del estructuralismo a partir de Lévi-Strauss. El mismo Bourdieu, heredero no siempre autoreconocido de esta tradición, señala que "lo real es relacional" (Bourdieu 2007:13) y recoge principios básicos de la tradición estructuralista en dos de sus conceptos matrices, campo y habitus y, de paso, como vimos al inicio de este artículo, utiliza el esquema cruzado, como representación gráfica del concepto de campo. Se infiere entonces que, de acuerdo a esta perspectiva, la constitución de la realidad social y cultural se fundamenta en la oposición y con ello en una relación entre elementos.

De ahí entonces también que la oposición graficada en los ejes respectivos del esquema cruzado representa a la vez una continuidad y una ruptura que lleva, por lo mismo, a la necesaria existencia de un concepto unificador, lo que desde Saussure, pasando por Lévi-Strauss y llegando a Bourdieu y a parte de la teorización sociológica actual, se condensa en el concepto de código. El código, definido binariamente, como la articulación de principios opuestos que refieren a una realidad mayor común, es decir, elementos que están diferenciados pero que, para hacerlo, necesitan un referente común y que solo pueden existir y estructurar el espacio social, en oposición. Un ejemplo clásico es el del referente "hombre" al que se le opone, inicialmente el de "mujer", ambos son tanto continuidad como opuestos y para existir en ambas 
dimensiones es absolutamente necesaria la referencia a un elemento mayor que el análisis estructural denominará eje semántico, en este caso "seres humanos". Este último será, por tanto, un código binario que encierra categorías polares, y que nos llevan al aspecto fundamental de todo análisis basado en la lógica estructural: los elementos codificados son comunicación encapsulada, que bajo una forma codificada o simbólica refieren a una externalidad evidente y a una internalidad subyacente o no consciente. La función del código es, precisamente, la de comunicar con eficacia. En esta línea explicativa, con todos los axiomas y argumentos señalados, se ubica el esquema cruzado y en particular las polaridades de cada uno de sus ejes darán cuenta de un código binario.

\section{Espacialidad}

Al tener una de sus bases en la geometría, las coordenadas cartesianas asumen como parte de su análisis a la dimensión espacial de objetos y relaciones estudiadas. ¿Recoge esto el esquema cruzado? En cierto sentido siempre lo hace, ya que una realidad al estar referida o ubicada en uno de los cuadrantes ocupa una posición en un plano. Sin embargo, existe otro elemento introducido por las coordenadas cartesianas que no siempre está presente en el esquema cruzado, y que se refiere a la espacialidad específica de un punto o fenómeno al interior del campo o cuadrante. Es lo que aparece en una de las figuras anteriores cuando se muestra que el punto $(4,3)$ está en una posición específica que lo diferencia relacionalmente de otros puntos, posiciones o fenómenos. De esta manera, un punto o fenómeno puede estar equidistante de ambos ejes o bien más cercano o lejano a una de ellos.

Esta espacialidad es finamente reconocida por la representación de campo que realiza Bourdieu, quien postula que los agentes tienen capitales y prácticas diferenciadas mientras más lejana es su oposición en el campo, así como lo contrario. La posición precisa que ocupan los agentes y sus prácticas culturales pasa a ser determinante en el esquema para precisar otro elemento central en la teorización del autor: las disposiciones de habitus.

Por su parte, Touraine et. al. (1981) señala algo similar. En los dos ejes presentados: clase/nación y defensa/modernización, los militantes analizados del movimiento occitano se encuentran posicionados diferenciadamente, algunos en el eje mismo en tanto otros en una posición equidistante. Tanto Bourdieu como Touraine recogen y explotan uno de los recursos epistémico-metodológicos centrales de las coordenadas cartesianas, la espacialidad, lo que no es el caso de otros de los autores revisados y de la metodología de análisis estructural propiamente tal. La estadística descriptiva e inferencial que trabaja con la gráfica de plano también ocupa la espacialidad de los puntos como un elemento central para conceptualizar los fenómenos.

\section{Fecundidad conceptual}

Un esquema o un constructo analítico es fecundo conceptualmente cuando los conceptos inicialmente identificados dan lugar, por combinatoria lógica, a nuevos conceptos. Esta idea está extraída del esquema cruzado utilizado en el análisis estructural de contenido, heredero directo de la tradición intelectual a la que hemos hecho referencia en las páginas anteriores. Los conceptos o realidades fecundadas son posibilidades lógicas y/o empíricas que reflejan la combinatoria de signos negativos y positivos de los cuadrantes de las coordenadas cartesianas, que aparece en los ejemplos de Brunner, Dubet y Ritzer y relativamente en el de Bourdieu. 
La fecundidad conceptual a partir del esquema cruzado parece ser uno de los aspectos más creativos e interesantes de este procedimiento. Por una parte permite pasar de cuatro conceptos polares (en los extremos de cada eje) a un total de ocho, sin considerar una exigencia de conceptos matrices que constituyan el eje semántico de la oposición señalada por cada línea, con lo cual se llegaría a diez conceptos. Lo interesante de esto es que produce, para el caso de la investigación empírica, la exigencia de probar la existencia factual de tales realidades. Pensemos en el siguiente ejemplo: un investigador propone un marco conceptual que graficado en el esquema cruzado establece un eje $X$ con la dualidad dictadura/democracia $y$ el eje Y con la oposición capitalismo/socialismo. De acuerdo a lo que hemos visto en este texto, estas relaciones de oposición parecen coherentes ya que es posible conceptualizarlas como oposiciones, es decir, como mutuamente excluyentes: en términos formales ( $y$ por supuesto no en términos sustanciales) democracia es una no dictadura y viceversa y capitalismo es un no socialismo, y viceversa. Ambas oposiciones refieren también a elementos comunes entre los términos, a ejes semánticos o códigos que parece ser regímenes políticos en el caso de $\mathrm{X}$ y tipos de economía en el eje de Y. Con ello el campo I aparece una combinatoria posible entre capitalismo y democracia, en el II entre capitalismo y dictadura, en el III entre dictadura y socialismo y en el IV entre socialismo y democracia. Ahora bien ¿qué nuevos conceptos es posible ubicar en cada campo? Recordemos que cada uno de estos conceptos (que grafican la "fecundación") son realidades hipotéticamente posibles, las que podrían ser:

Campo I: Democracia liberal

Campo II: Dictaduras con régimen de mercado

Campo III: Socialdemocracia

Campo IV: Socialismos reales

El resto pasa a ser, como en todo trabajo de investigación, la exigencia de comprobación de estas hipótesis y de ser posible, la espacialización precisa de realidades empíricas concretas.

Considerando los ejemplos de autores del inicio de este artículo y las tres características señaladas en la epistemología del esquema cruzado, podemos establecer la siguiente presencia de las mismas en los textos referidos, sin que ello significa falencias ni virtudes, sino solo una relación epistemológica más estricta con el origen del esquema en cuestión. Se observa en todo caso que el elemento utilizado por todos los autores es la lógica de opuestos y que el único autor que toma los tres aspectos reseñados es Bourdieu:

\begin{tabular}{|l|c|c|c|}
\hline \multicolumn{1}{|c|}{ Autor/Dimensión } & Lógica de opuestos & Espacialidad & Fecundidad conceptual \\
\hline Touraine & Sí & No & No \\
\hline Touraine et al & Sí & Sí & No \\
\hline Dubet & Sí & No & Sí \\
\hline Brunner & Sí & No & Sí \\
\hline Ritzer & Sí & No & Sí \\
\hline Bourdieu & Sí & Sí & \\
\hline
\end{tabular}

\section{Conclusiones}

El análisis de constructos metodológicos tales como el esquema cruzado debe ser considerado al interior de un programa más amplio de investigación que se relacione con el estudio de la esquematización y la 
formalización en la investigación social. Los esquemas formales son instrumentos analíticos que no son mayormente utilizados en la investigación cuantitativa, puesto que esta tiene su propia expresión esquemática a partir de la formalización matemática. Precisamente, el aporte de la esquematización a la investigación cualitativa es el de la formalización de una parte o de la totalidad de los procedimientos de formulación del análisis y de la validación empírica de los resultados. La pregunta es, entonces, ¿por qué es necesaria la formalización en ciencias sociales y cuáles son los requerimientos de la misma en el campo de investigación conceptual o empírica pero de base cualitativa? En segundo lugar cabe preguntarse cuáles son los requerimientos de un análisis de pretensiones formales en investigación social cualitativa y si la perspectiva de análisis aquí presentada cumple con tales requisitos.

Respecto de la primera pregunta, la formalización de los procedimientos de análisis puede o no ser necesaria, dependiendo del nivel de conocimiento del objeto de estudio y por supuesto de la perspectiva epistemológica y de programa de investigación en la cual se sitúe el investigador. La formalización necesariamente requiere de una perspectiva de validación del conocimiento a partir de una estandarización del procedimiento. El elemento a favor que cuenta todo proceso de estandarización, tal como lo hemos visto en este artículo, es que o bien alude explícitamente a una perspectiva teórica o bien deja en evidencia las contradicciones (al menos parciales) con la misma.

En relación a la segunda pregunta, un análisis de pretensiones formales tiene, precisamente, la exigencia de explicitación teórica del mismo o, dicho de otra manera, la demostración de la coherencia entre la cadena de presupuestos epistemológicos, elementos teóricos y constructos metodológicos para abordar la realidad en estudio. Tal fue durante décadas la ventaja de la perspectiva estructuralista, aunque también es necesario mencionar otros programas de investigación como la antropología cognitiva o etnociencia que también tiene relación con la lingüística estructural y, en menor grado, de la teoría fundada (grounded theory) cuya máxima prueba de formalización ha sido la de generar un software (Atlas-ti) adecuado a sus procedimientos de análisis.

Finalmente nos parece importante remarcar que aun cuando el esquema cruzado proviene de las coordenadas cartesianas y de la tradición estructuralista en lingüística y ciencias sociales, su uso se vincula, como lo vimos al comienzo del texto, con tradiciones variadas en ciencias sociales. Aun así parece claro que en cada una de sus utilizaciones se mantiene, en mayor o menor grado, una vinculación con su epistemología de base, que emana de las tradiciones mencionadas.

\section{Nota}

(1) Artículo desarrollado como parte de la docencia regular en el Programa de Magister en Política Educativa de la Universidad Alberto Hurtado, cátedra de Técnicas cualitativas parta el análisis de políticas educacionales.

(2) Para conservar la presentación gráfica original de los autores hemos trasladado los esquemas manteniendo, cuando correspondía, los términos en francés, considerando que la cercanía escrita de esta lengua al español permite una fácil comprensión.

\section{Bibliografía}

Barthes, R. 1971. Elementos de semiología. México: Alberto Corazón editor. 
Bourdieu, P. 1979. La distinction. Paris: Minuit.

Bourdieu, P. 2007. Razones prácticas. Barcelona: Anagrama.

Brunner, J.J. 1994. Cartografías de la modernidad. Santiago: Dolmen.

Brunner, J.J. 1998. Globalización, cultura y posmodernidad. Santiago: Fondo de Cultura Económica.

Brunner, J.J. 2000. Universidad siglo XXI América Latina: regulación y financiamiento. Estudios Sociales 106: 39-73.

Descartes, R. 1858. La géométrie. Paris: A. Hermann librairie scientifique.

Dubet, F; Tironi, E; Espinoza, V. y Valenzuela, E. 1989. Pobladores. Paris: L`Harmattan.

Greimas, A. 1971. Semántica estructural. Buenos Aires: Losada.

Hiernaux, J.P. 1977. L'Institution culturelle. Louvain: Presse Universitaires de Louvain.

Lévi-Strauss, C. 1974. Anthropologie structurale. Paris: Agora.

Piret, A; Nizet, J. y Bourgeois, E. 1996. L'analyse structural. Bruselas: De Boeck.

Rémy, J. 1990. L'analyse structurale et la symbolique sociale. En: Rémy, J. y Ruquoy, D. (Eds). Méthode d'analyse de contenue et sociologie. Bruxelles: Publications des Facultés Universitaire Saint-Louis, pp. 93109.

Ritzer, G. 1996. Teoría sociológica contemporánea. México: McGraw Hill.

Touraine, A. 1978. La voix et le regard. Paris: Du Seuil.

Touraine, A; Dubet, F; Wieviorka, M. y Hegedus, Z. 1981. Le pays contre I'Etat. Paris: Seuil

Recibido el 18 May 2011

Aceptado el 24 Ago 2011 